\title{
Dejar de fumar precozmente durante el embarazo reduce las tasas de nacimiento pretérmino y de bajo peso para la edad gestacional
}

Early stop smoking during pregnancy reduces the incidence of spontaneous preterm birth and small for gestational age infants

Mc Cowan L, y col. BMJ 2009;338:b1081doi:10.1136/bmj.b1081

\section{Objetivo}

Comparar las tasas de nacimiento pretérmino y de bajo peso para la edad gestacional (BPEG) en mujeres que dejaron de fumar antes de las 15 semanas de gestación contra aquellas que continuaron fumando, y contra quienes no fumaron durante el embarazo.

\section{Diseño}

Estudio prospectivo de cohortes.

Lugar

Auckland, Nueva Zelanda; y Adelaida, Australia.

\section{Pacientes}

Se reclutaron 2504 mujeres sanas, nulíparas y con embarazo único a las 15 semanas de gestación.

\section{Evaluación de los factores de riesgo}

Se las dividió en tres grupos, según el status de fumadoras

reportado por las participantes: 1) no fumadoras ( $\mathrm{n}=1992)$; 2$)$ las que dejaron de fumar antes de las 15 semanas $(n=261) ; 3)$ las que continuaron fumando luego de las 15 semanas $(n=251)$. Tuvieron un seguimiento prospectivo, completo en el $99 \%$ de las participantes.

\section{Medición de los resultados principales}

Tasas de nacimiento pretérmino y de BPEG.

\section{Resultados principales}

Luego de ajustar por confundidores* potenciales (edad, grupo étnico, estado marital, empleo, índice de masa corporal, sangrado durante el embarazo, uso de ácido fólico, consumo de alcohol, niveles de depresión, ansiedad y estrés) no se observaron diferencias significativas en las tasas de nacimiento pretérmino o de BPEG entre las mujeres que dejaron de fumar y aquellas no fumadoras. En cambio, comparado con las mujeres que dejaron de fumar, aquellas que continuaron fumando presentaron tasas significativamente más altas de los dos eventos de interés (ver tabla 1).

Tabla 1: riesgo de nacimiento pretérmino y BPEG de acuerdo al hábito tabáquico.

\begin{tabular}{c|c|c|c|c}
\hline Resultado de interés & \multicolumn{2}{|c|}{ RA según hábito tabáquico } & OR ajustado (IC95\%) & NND \\
\hline \multirow{2}{*}{ Nacimiento pretérmino } & No fumadoras $(\mathrm{n}=1992)$ & $4 \%$ & $1,03(0,49$ a 2,18) & - \\
\cline { 2 - 5 } & Fumadoras $(\mathrm{n}=251)$ & $10 \%$ & $3,21(1,42$ a 7,23$)$ & 16 \\
\hline $\begin{array}{c}\text { Bajo peso para la edad } \\
\text { gestacional }\end{array}$ & No fumadoras $(\mathrm{n}=1992)$ & $10 \%$ & $1,06(0,67$ a 1,68) & - \\
\cline { 2 - 5 } & Fumadoras $(\mathrm{n}=251)$ & $17 \%$ & $1,76(1,03$ a 3,02$)$ & 14 \\
\hline
\end{tabular}

BPEG: bajo peso para la edad gestacional. RA: Riesgo absoluto. OR: odds ratio*. NND: número necesario para dañar*.

\section{Conclusiones}

Las mujeres que dejan de fumar antes de de las 15 semanas de gestación reducen el riesgo de nacimiento pretérmino y de BPEG con respecto a las que continúan fumando e igualan el mismo con respecto a las no fumadoras.
Palabras claves: tabaquismo, bajo peso para la edad gestacional, nacimiento pretérmino. Keywords: smoking, spontaneous preterm birth, small for gestational age.

Fuentes de financiamiento: New Enterprise Research Fund, Foundation for Research Science and Technology; Health Reserch Council; Evelyn Bond Fund, Auckland District Health Board Charitable Trust; Premier's Science and Research Fund, South Australian Government

\section{Comentario}

Aunque es difícil conocer la prevalencia exacta del tabaquismo durante el embarazo, se estima que ésta alcanza al $14 \%$. Fumar es el factor de riesgo modificable más importante asociado con eventos adversos del embarazo. Se sabe que existe una relación causal y dosis dependiente entre el fumar durante el embarazo y el riesgo de tener un nacimiento pretérmino y un recién nacido con $\mathrm{BPEG}^{1}$, con tasas que alcanzan un 10 y $30 \%$, respectivamente ${ }^{2}$.

Como se vio en este estudio, fumar durante el embarazo se asocia a otros factores de riesgo de eventos adversos perinatales, como ser: la edad menor de 25 años, un bajo nivel de escolaridad e ingresos, la soltería y el mayor consumo de alcohol. Esto es consistente con lo hallado en otras publicaciones. Debido a la heterogeneidad entre los grupos relacionada con los factores previamente mencionados se debió realizar un análisis ajustado de los datos.
En este trabajo las mujeres que continuaron fumando tuvieron tasas significativamente más altas de eventos adversos que las que dejaron de fumar. Si bien los intervalos de confianza son amplios, la dirección del efecto no sólo es estadísticamente significativa, sino también clínicamente relevante. Es probable que un estudio con mayor número de pacientes mejore la precisión del efecto de la cesación tabáquica pero no modifique los resultados observados.

\section{Conclusión del comentador}

Los resultados de este estudio deben motivarnos aun más a incentivar el cese tabáquico en las pacientes que continúan fumando en el primer trimestre de gestación.

Catalina Brosens [ Servicio de Medicina Familiar y Comunitaria del Hospital Italiano de Buenos Aires catalina.brosens@hospitalitaliano.org.ar ]

Brosens C. Dejar de fumar precozmente durante el embarazo reduce las tasas de nacimiento pretérmino y de bajo peso para la edad gestacional. Evid Act Pract Ambul. 13(2) 53. Abr-Jun 2010. Comentado de Mc Cowan L y col. Spontaneous preterm Birth and small for gestational age infants in women who stop smoking early in pregnancy: prospective cohort study. Mc Cowan L, y col. BMJ 2009;338:b1081doi:10.1136/bmj.b1081. PMID 19325177. 